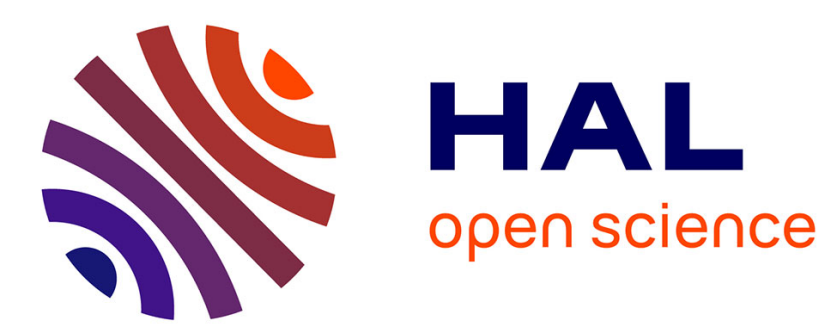

\title{
Discrete time-frequency (time-scale) techniques for time delay and Doppler estimation
}

\author{
Abdeldjalil Ouahabi, Denis Kouamé
}

\section{To cite this version:}

Abdeldjalil Ouahabi, Denis Kouamé. Discrete time-frequency (time-scale) techniques for time delay and Doppler estimation. 6th IEEE International Conference on Electronics, Circuits, and Systems (ICECS 1999), Sep 1999, Paphos, Cyprus. pp.1457-1460, 10.1109/ICECS.1999.814444 . hal03153369

\section{HAL Id: hal-03153369 \\ https://hal.science/hal-03153369}

Submitted on 1 Mar 2021

HAL is a multi-disciplinary open access archive for the deposit and dissemination of scientific research documents, whether they are published or not. The documents may come from teaching and research institutions in France or abroad, or from public or private research centers.
L'archive ouverte pluridisciplinaire HAL, est destinée au dépôt et à la diffusion de documents scientifiques de niveau recherche, publiés ou non, émanant des établissements d'enseignement et de recherche français ou étrangers, des laboratoires publics ou privés. 


\title{
DISCRETE TIME-FREQUENCY (TIME-SCALE) TECHNIQUES FOR TIME DELAY AND DOPPLER ESTIMATION
}

\author{
A. Ouahabi, Member, IEEE, and D. Kouamé, Member, IEEE \\ GIP ULTRASONS/LUSSI. \\ 2 bis boulevard Tonnellé, BP 3232, 37023 TOURS Cédex 3, FRANCE. \\ E-mail: ouahabi@univ-tours.fr
}

\begin{abstract}
This paper is focused on joint fine estimation of time delay and Doppler shift based on the maximum likelihood criteria. Joint estimation of time delay and Doppler stretch of a random signal received by two (or more) sensors is required in many signal processing applications. This problem can be viewed as the determination of the effects of a time-varying channel on the emitted signal. It is shown that discrete timefrequency (time-scale) techniques provide the correct system characterization. Theses techniques are simple, fast and efficient. Moreover, in areas where the measurements are repeatable, such as ultrasonic imaging and nondestructive evaluation, the process of averaging can be used to improve the SNR.

In order to implement the simultaneous estimation of time delay and Doppler coefficient according to the maximum likelihood criterion, two practical problems are investigated: the interpolation method used to obtain a fine resolution of the estimates and the scaling procedure which is not a trivial problem.
\end{abstract}

\section{INTRODUCTION}

The problem of estimating the time delay and Doppler shift of a known waveform is important in two common applications. First, in active radar and sonar, a known waveform is transmitted, and reflections from objects "illuminated" by the transmission are subsequently received. The received signals are often modeled as scaled, delayed and Doppler-shifted versions of the transmitted signal. Estimation of the signal amplitude, delay, and Doppler shift provides information about the position and relative motion of the objects.

The second application involves estimation of the parameters of a multi-path communication channel in situations where the transmitter is rapidly moving or has an unknown frequency offset. For a example, consider a situation where a remote mobile user transmits a known waveform (e.g., a training sequence) to a base station for synchronization or equalization purposes. If the channel is frequency selective (nonzero delay spread), then the signal will be received with several distinct delays. In addition, due to the motion of the mobile and variations in the carrier frequency of the transmitter, the known signal can be received with a small frequency offset. Estimation of the delays and frequency offsets, as well as the spatial signatures of the signal arrivals, is necessary in establishing a clean, inter-symbol, and interference-free communication link.

The interest in time delay estimation has moved from the initial area of radar and underwater acoustics. In particular, digital methods for delay and Doppler fast estimation are very important in medical application such as blood velocity which is an important parameter for the clinical diagnosis of vascular disease. Ultrasound has become an indispensable noninvasive tool for blood velocity measurement. The pulsed wave Doppler technique is widely used because it can provide range resolution. With this method, sequential short ultrasound pulses are transmitted into vessel or heart at a pulse repetition frequency. Return signals are received sequentially after a certain delay following the pulse transmission. Due to the movement of the scatterers, the received echo is a time-delayed version of the echo from the previous pulse if time transit effects are not considered.

Classical approaches of time-delay and Doppler estimation are based on matched filtering. These techniques typically assume one signal path and one sensor, although the extension to multiple sensor is straightforward. Matched filtering techniques are known to be optimal in the maximum likelihood (ML) sense for a signal arrival (corrupted by additive, Gaussian, white noise) but are not consistent when multiple overlapping copies of the signal are present.

Digital processing techniques, based on fast interpolation of some estimated ambiguity samples, are particularly suited for real-time estimation of signal parameters to reduce the tremendous computational cost of a 2-D processing. They are based on parabolic interpolation of the sampled cross-correlation function (CCF) or cross ambiguity or optimum discrete wavelet scaling.

This paper is focused on joint fine estimation of time delay and Doppler coefficient by a fast interpolation of the estimated ambiguity function, and used a new and computationally efficient solution to the scaling problem.

The outline of the paper is as follows. In the next section, the signal model is described. Section 3, presents the ML solution of time delay and Doppler estimation. Finally, Section 4 provides a 
computationally efficient solution to the interpolation and scaling problem.

\section{MODELING AND HYPOTHESIS}

Let us refer to the following model of signals

$$
\begin{aligned}
& x(t)=s(t)+n(t) \\
& y(t)=s_{\theta_{o}}(t)+m(t)
\end{aligned}
$$

received by two sensors:

where $s(t)$ is the source signal, and the noises $n(t)$ and $m(t)$ are uncorrelated with each other and with the signal $s(t)$.

The received signal is expected to take one of two form:

$$
\begin{aligned}
& s_{\theta_{0}}(t)=A s(t-d) e^{j \omega_{D} t} \text { for narrowband signals } \\
& s_{\theta_{0}}(t)=A s((t-d) / a) \text { for wideband signals }
\end{aligned}
$$

$\begin{array}{ll}\text { where } & \\ \omega_{D} & \text { Doppler shift; } \\ A & \text { attenuation; } \\ D & \text { time difference of arrival; } \\ a & \text { scale difference of arrival. }\end{array}$

A correlation processor or a matched filter combines the transmitted signal $s(t)$ (or $x(t)$ in additive noise) with the received signal $s_{\theta o}(t)$ (or $y(t)$ in additive noise). A high correlation results when the signals match. This processing technique is optimal for signals buried in additive, Gaussian, white noise [9]. The correlation or the matching operation requires some form of model for the received signal, two of which are the narrow-band model and the wide-band model. The model selected depends on the assumed characteristics of the received signal. Suppose a transducer emits the signal $s(t)$, and this signal reflects off a single object (a point scatterer). If the object moves with constant velocity, $v$, a time scaling, $a$, of the signal occurs. The received signal is then modeled as a wide-band signal model.

When the speed of propagation of the signal, $c$, verify $|v|<c$, the wide-band model can be approximated by narrow-band model [3]. With this model, time scaling the signal by $a$ is approximated by a Doppler shift, $\omega_{D}$. For a signal with carrier frequency, $\omega_{c}=2 \pi f_{c}$, the approximation is

$\omega_{D} \equiv \frac{-2 v}{c} \omega_{c} \approx\left(\frac{1-a}{a}\right) \omega_{c}=(\eta-1) \omega_{c}$

with $\eta=\frac{1}{a}$.

This approximation says that all frequencies in the signal are shifted equally. The approximation is based on the assumption that for signals with a sufficiently narrow bandwidth, time-scaling the signal is equivalent to a frequency shift.

Active echo location (or time-varying system characterization) techniques seek to identify and localize objects in an environment [7]. One way to accomplish this is to estimate values of $\tau$ and $a$ (or $\omega_{p}$ ) associated with each object [6]. Various techniques have been developed to estimate theses parameters, with one commonly applied technique being correlation processing.

\section{TIME-FREQUENCY (TIME-SCALE) PROCESSING}

The idea behind correlation processing is to correlate the received signal, $s_{\theta o}(t)$, with hypothesized replicas of the transmitted signal, $s(t)$, where a collection of delays and scales (Doppler shifts) are hypothesized. When the received signal and one of these hypothesized signal match, a high correlation results. The hypothesized signal yielding the highest correlation then provides an estimate of the parameters of the received signal.

The correlation processing is then:

1. Wide-band model (time-scale analysis)

$W(a, \tau)=\frac{1}{\sqrt{a}} \int_{-\infty}^{+\infty} s_{\theta_{o}}(t) s *\left(\frac{t-\tau}{a}\right) d t$

Expression (4) represents a wide-band correlation receiver output or the wide-band cross ambiguity function of the signals $s(t)$ and $s_{\theta o}(t)$ and it is referred to as a continuous wavelet transform of the signal $s_{\theta o}(t)$ with respect to the mother wavelet, $s(t)$.

2. Narrow-band model (time-frequency analysis)

$A\left(\omega_{D}, \tau\right)=\int_{-\infty}^{+\infty} s_{\theta_{o}}(t) s *(t-\tau) k^{-j \omega_{D} t} d t$

Expression (5) represents the narrow-band cross ambiguity function of the signals $s(t)$ and $s_{\theta o}(t)$.

$C(\tau)=\int_{-\infty}^{+\infty} s_{\theta_{o}}(t) s *(t-\tau) d t$

When the transmitter/receiver is motionless and the signal, $s(t)$, is reflected from a motionless object, then $a=1$ and $\omega_{\mathrm{D}}=0$. In this case, both Eqs (4) and (5) reduce to the same equation:

This equation is the standard correlation encountered in time delay estimation theory [1].

Since, the dominant effect of relative motion consists of a time scaling of the received signal, we focus on system characterization with the wide-band model.

In this case, the time delay $\hat{d}$ and time scale a estimates are

$(\hat{a}, \bar{d})=\arg \max _{a, \tau}\{W(a, \tau)\}$

The optimum wavelet in this case is identified as one of the sensor output [4]. 
Previous papers did not explicitly use discrete-time representations. In fact, continuous-time implementation of Doppler time scaling is quite complex and expensive. Moreover, all the possible Doppler scaling factor should be considered in the maximum likelihood procedure.

Digital processing techniques, based on fast interpolation of some estimated ambiguity samples, are particularly suited for real-time estimation of signal parameters to reduce the tremendous computational cost of 2-D processing. In practice, the signal is discrete due to sampling in time. The true location of the maximum of a 2-D ambiguity function is not constrained to discrete increments, and it may fall between the discrete sampling points that result in estimation inaccuracy. An interpolation technique usually is used to improve the time delay and Doppler estimation accuracy. The most widely used interpolation technique is the simple parabolic-fit [8].

\section{THE SCALING \& INTERPOLATION PROBLEMS}

In practice, only the low-passed signal samples are available. Now, expression (4) in discrete form reads (ignoring $1 / \sqrt{a}$ )

$W(a, \tau)=\sum_{k=-N^{\prime}}^{k=N^{\prime}} y(k) x_{1, a}(k-\tau)$

With $N=2 N^{\prime}+1$ noisy data samples.

The estimation procedure is to scale $x_{1}(k)$ over several values and, for each scale, to compute the crosscorrelation as in (8). The pair $(a, d)$ that gives maximum correlation value is the final estimate. The FFT is used to calculate the convolution in (8).

Several approaches have been proposed for the scaling of a wavelet that has no analytic form. One method is by multi-rate signal processing [2]. It involves up-sampling, filtering, and down-sampling the input sequence. If the scaling factor is close to unity, the multi-rate method needs large up-sampling and down-sampling factors, giving rise to high complexity [10]. Recently, Young [11] has proposed another technique by using the cross wavelet transform [10], [11]. The technique first transforms the discrete signal by an admissible analytic wavelet over sufficient scales and shifts, and applies then applies the wavelet inversion formula to compress or expand the signal. The method is computationally intensive as a large number of shifts and scales are needed for good accuracy.

Therefore it is not trivial to scale $x_{1}(k)$ since it has no analytic form. Scaling via the multi-rate technique is very costly and prohibits real-time application. Although incoherent processing is computationally attractive, it can only give sub-optimal solutions. We shall use the scaling method proposed by Ho and Chan [5] and demonstrate its effectiveness in the estimation problem.

\subsection{Fast scaling algorithm}

Ho and Chan [5] have formulated the scaling problem as a mean-square minimization, and the resulting estimator consists of two parts: noise filtering and sinc function scaling. Sinc function scaling is a time-dependent process and requires $O\left(N^{2}\right)$ operations, where $N$ is the data length. The fast algorithm based on the FFT is proposed to reduce the complexity to $O\left(N \log _{2} N\right)$.

The scaling problem can be stated as follows: Given $N=2 N^{\prime}+1$ noisy data samples

$x(k)=s(k)+n(k), \quad-N^{\prime} \leq k \leq N^{\prime}$

we want to find $s_{a}(k)=s(t / k)_{t=k}$ which are the signal samples scaled by a factor of $a$. The sampling time is normalized to unity, and $n(k)$ is the noise. The number of samples of the scaled signal may be more or less than $N$, depending on the scaling factor $a$. Here, we set the scaled signal to have the same number of samples as the original signal. In such case, for $a<1$ (compression), the extra samples are from extrapolating $s_{a}(t)$, whereas for $a>1$ (expansion), the end samples of $s_{a}(t)$ are not considered.

The estimate of $s_{a}, \hat{s}_{a}$ can be expressed as $\mathbf{H x}$ where $\mathbf{H}$ is an $N \times N$ matrix. The mean square error is an optimal criterion to obtain the operator $\mathbf{H}$. Operating $\mathbf{H}$ on $\mathbf{x}$ can be expressed in two steps:

$\widehat{\mathrm{s}}=\mathrm{R}_{s s} \mathrm{R}_{x x}^{-1} \mathrm{x}$ noise filtering (Wiener filtering)

$\overline{\mathrm{s}}_{a}=\mathrm{A}_{a} \overline{\mathrm{s}} \quad$ scaling

where $A_{a}$ is a pure scaling operator and $\mathrm{R}_{x x}=E\left\{\mathrm{xx}^{T}\right\}=\mathrm{R}_{s s}+\mathrm{R}_{n n}$ because signal and noise are independent.

Expression (11) can be expressed as a fast convolution (based on FFT):

$\bar{s}_{a}(k)=\frac{1}{N} e^{j \frac{\pi}{a N} k^{2}} \sum_{i=-N^{\prime}}^{i=N^{\prime}} \alpha(i) \beta^{*}(k-i)$

where $\alpha(i)=\bar{S}(i) e^{j \frac{\pi}{a N} i^{2}}$ and $\beta(i)=e^{j \frac{\pi}{a N} i^{2}}$

$\widehat{S}$ is the Fourier transform of $\widehat{s}$, defined in the limited

range $|i| \leq B N / 2$ with $B= \begin{cases}1 & a>1 \\ a & a<1\end{cases}$

Assuming the sequence $\beta(i)$ and its FFT are available in memory, the required complex computation in each scale is

1) N operations for $\alpha(i)$;

2) 2 length $(2 N-1)$ FFTs and $N$ operations for convolving $\alpha(i)$ and $\beta(i)$;

3) $2 N$ operations to multiply the convolution sum by $\beta(i)$ and $N(N$ operations only if $\beta(i) / N$ are precomputed). 
The FFT of input $\hat{s}$ is needed only once and is not counted. Since convolution dominates the computational load, the expense to perform one discrete signal scaling is the order of $4 N \log _{2}(2 N)$ complex operations. The reduction in complexity is significant when is $N$ large.

\subsection{Parabolic interpolation}

In a more general case, if the functions $W(a, \tau)$ have a convex form in a neighborhood of its maximum, a local interpolation is necessary to get a good time delay and Doppler estimation. One simple interpolation method is parabolic-fitting, which has been used in many applications.

In our application, the parabolic fitting is performed near the peak and only requires a few operations. This local interpolation can be accomplished by a fast technique by determining a 2-D paraboloid

$W(a, \tau) \approx \alpha \tau^{2}+\beta a^{2}+\gamma \tau+\delta a+\varepsilon$

fitted from only five estimates (five measurements of $W(a, \tau)$ around its maximum). It should be noted that such an approximate fast interpolation is here very important to reduce the tremendous computational cost of searching for the minimum over the whole 2-D domain. The time delay and time scale can be performed as

$\hat{d}=\frac{-\gamma}{2 \alpha} \quad$ and $\quad \hat{a}=-\frac{\delta}{2 \beta}$

Unfortunately, the parabolic-fit interpolation method introduces a bias at low sampling rate. It is interesting to use other methods as linear filtering to reduce this bias.

\section{CONCLUSIONS}

The time delay and Doppler estimation is examined on two data sets for simulated and real backscattered signals. Different experimental conditions: multiple and non-multiple time delay and time scale of the sampling rate under different signal-to-noise ratio (SNR's) will be presented at ICECS'99. We begin by studying the performance of the algorithms presented above as the signal-to-noise ratio (SNR) varies ( -10 to $30 \mathrm{~dB}$ ). Simulation data was generated using (1) with time delays $d=0.5$ and $d=2$, and time-scale $a=0.988$ and $a=1.011$. The data was corrupted by white Gaussian noise with zero mean and standard deviation $\sigma$. The signal sequence was chosen to be

$s(k)=\left(\frac{k}{3 M}\right) \sin \left(2 \pi f_{0} k\right) e^{\left(3-\frac{t}{M}\right)}$

where the time $k$ varies form 0 to 100 , and $M$ is a constant.

An other signal considered next is a correlated $\operatorname{AR}(2)$ signal

$s(k)=1.16 s(k-1)-0.81 s(k-1)+\varepsilon(k)$ where $\varepsilon(k)$ is a Gaussian white sequence of power $\sigma^{2}$

The bias and standard deviation of the estimators are performed on 100 realizations.

Experimental evaluation is performed in ultrasound domain oriented to source localization and channel modeling and system characterization (blood vessel) in high signal-to-noise situations.

In this paper, we have developed a data model for time delay and Doppler estimation problem. We have revisited the general solution of this problem using the maximum likelihood criterion. Our study is focused on the scaling problem and the necessity of interpolating. The algorithm proposed can be used also when the signal $s(t)$ is random or a filtered noise (AR signal).

Preliminary results are very encouraging step for using the fast algorithms studied.

\section{ACKNOWLEDGMENT}

The authors would like to thank Prof. Y. T. Chan for useful comments in a private correspondence concerning the scaling problem.

\section{REFERENCES}

[1] G. C. Carter and P. B. Abraham, "Estimation of source motion from time delay and time compression," $J$. Acoust. Soc. Amer., vol. 67, N0 3, pp. 830-832, March 1980.

[2] R. E. Crochiere and L. R. Rabiner, Multirate Digital Signal Processing, Prentice-Hall, New Jersey, 1983.

[3] T. H. Glisson, C. I. Black and A. P. Sage, "On sonar signal analysis," IEEE trans Aero.. Elect. Systems, vol. 6, N0 2, pp. 37-49, Jan. 1970.

[4] C. Guetbi, D. Kouamé, A. Ouahabi, J. P. Chemla , "Methods based on wavelets for time delay estimation of ultrasound signals," Lisbon, Portugal, Sept. 1998, pp. 113-116.

[5] K. C. Ho and Y. T. Luo, " Optimum discrete scaling and its application to delay and Doppler estimation," IEEE trans. Signal Processing, vol. 46, pp. 904-916, April 1995.

[6] Q. Jin, K. M. Wong and Z. Q. T. Chan, " The estimation of time delay and Doppler stretch of wideband signals," IEEE trans. Signal Processing, vol. 43, N0 9, pp. 22852290, Sept. 1998

[7] C. H. Knapp and G. C. Carter, "Estimation of time delay in the presence of source or receiver motion," J. Acoust. Soc. Amer., vol. 61, N0 6, pp. 1541-1549, June 1977.

[8] X. Lai and H. Torp, "Interpolation methods for timedelay estimation using cross-correlation method for blood velocity measurement," IEEE trans. Ultrasonics, Ferroelctrics and Freq. Control, vol. 46, N0 2, pp. 276289, March 1999.

[9] H. Van Trees, Detection, estimation, and modulation, Wiley, New Jersey, 1968.

[10] L. G. Weiss, "Wavelets and wideband correlation processing", IEEE Signal Processing Magazine, pp. 1332, Jan. 1994.

[11] R. K. Young, Wavelet and its Applications, Kluwer Academic Publishers, Boston, 1993. 